\title{
Séance du Comité central du 21 septembre 2012
}

\begin{abstract}
Factures hospitalières - Le Comité central (CC) est favorable au fait que les factures DRG ne contiennent strictement que les indications déterminantes pour le groupage. Or le projet soumis par santésuisse et $\mathrm{H}+$ à l'OFSP et repris dans le projet d'ordonnance ne respecte pas cette limitation et c'est pourquoi le CC le rejette. Vous trouverez de plus amples informations à ce sujet dans l'éditorial du $\mathrm{n}^{\circ} 43$ du Bulletin des médecins suisses.
\end{abstract}

Documents d'application relatifs aux HTA - SwissHTA est en train d'élaborer trois documents d'application concernant l'institutionnalisation et la méthodologie des HTA (Health Technology Assessment) en Suisse. Le CC les approuve malgré quelques divergences de vue sur des questions de détail et se déclare favorable à la variante visant la création d'une institution HTA de droit public.

Enquête sur la médecine de famille - D'avril à juin 2012, le Commonwealth Fund a réalisé une enquête sur la médecine de famille dans différents pays et notamment auprès de 1000 médecins de premier recours en Suisse. En collaboration avec Médecins de Famille Suisse, le CC a pu inclure dans cette enquête quelques questions spécifiques à la médecine de premier recours suisse. Les résultats seront présentés en novembre à Washington et paraîtront ensuite dans le Bulletin des médecins suisses.

Conseils aux femmes enceintes - Dans le cadre du programme national "Alcool/PNA», il s'agira, en s'appuyant sur une base scientifique, de trouver un consensus pour fournir un soutien aux femmes enceintes concernant les risques liés à la consommation d'alcool. La FMH s'engage depuis les années 90 dans les programmes de prévention fédéraux et collabore étroitement avec Addiction Suisse. Il s'agit maintenant d'actualiser la brochure «Alcool et grossesse» afin de garantir l'unité des informations destinées aux médecins de famille, aux gynécologues et aux centres spécialisés dans les dépendances.

\section{Lafffliation à la FMH n'a que des avantages.}

\author{
Nous vous soutenons grâce à une politique \\ engagée et des services attrayants: ligne \\ d'assistance tarifaire, renseignements juri- \\ diques, contrats-type.
}

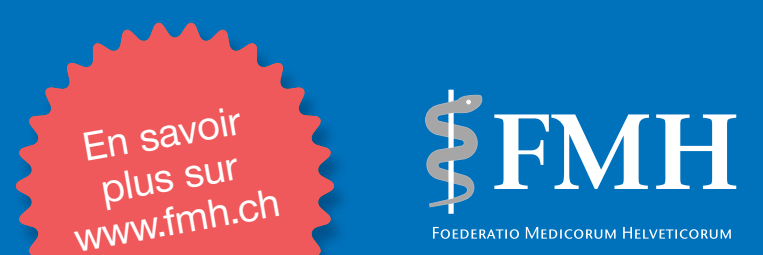

